\title{
Correlation between Preoperative Magnetic Resonance Imaging Signal Intensity Changes and Clinical Outcomes in Patients Surgically Treated for Cervical Myeloradiculopathy
}

\author{
Chaitanya Baban Chikhale, Ketan Shripad Khurjekar, Ashok Kumar Shyam, Parag Kantilal Sancheti \\ Department of Orthopaedics, Sancheti Institute for Orthopaedics and Rehabilitation, Pune, India
}

Study Design: This was a single surgeon, single center-based retrospective study with prospective data collection.

Purpose: To assess the correlation between T2-weighted magnetic resonance imaging (MRI) signal intensity (SI) changes and factors such as age, duration of symptoms, baseline modified Japanese Orthopedic Association (mJOA) score and to determine its prognostic value in predicting recovery after surgery.

Overview of Literature: Whether intramedullary cord T2-weighted MRI SI changes can predict operative outcomes of cervical myeloradiculopathy remains debatable, with only a few prospective studies analyzing the same.

Methods: Forty-six consecutive patients who underwent cervical myeloradiculopathy were included and were followed up for an average of 1 year. Preoperative T2-weighted MRI SI grading was performed for all patients. The correlation between MRI SI changes and age, duration of symptoms, preoperative mJOA score, and mJOA score at 1-year follow-up were analyzed.

Results: Fifteen patients had single-level (21.73\%) or double-level (10.86\%) prolapsed discs; $54.34 \%$ had degenerative cervical spondylosis with canal stenosis or multilevel disc prolapse and $13.07 \%$ had ossified posterior longitudinal ligaments. The mean age was $56.17 \pm 9.53$ years (range, 35-81 years). The mean baseline mJOA score was $10.83 \pm 2.58$ (range, $6-16$ ), which postoperatively improved to $13.59 \pm 2.28$ (range, $8-17 ; p<0.001$ ). There was a statistically significant correlation between mJOA score at 1 year and MRI T2 SI grading $(p=0.017)$.

Conclusions: Patients with longer symptom durations had high grades of intramedullary cord T2-weighted MRI SI changes. Age and preoperative neurological status were not significantly correlated with the existence of intramedullary cord SI changes. However, patients without or with mild and diffuse intramedullary cord T2-weighted MRI SI changes had better postoperative neurological recovery than those with sharp and focal SI changes.

Keywords: Cervical myelopathy; Magnetic resonance imaging; Intramedullary spinal cord signal intensity; Neurologic outcome; Prognosis

\section{Introduction}

The natural history of cervical myeloradiculopathy sug- gests that this condition is progressive in nature and is bound to result in neurological decline. Consequently, many of these patients require surgical intervention [1].

\footnotetext{
Received May 17, 2016; Revised Jul 19, 2016; Accepted Jul 25, 2016

Corresponding author: Chaitanya Baban Chikhale

Sancheti Institute for Orthopaedics and Rehabilitation, Shivajinagar, Pune, India [411005].

Fax: +98-3311-0366, E-mail: chaitanya14486@gmail.com
} 
Considering that many patients with myeloradicluopathy may improve even without any surgery increases the importance of prospectively determining which patients are likely to benefit from decompression surgery [2].

Patients scheduled for decompression surgery should undergo preoperative magnetic resonance imaging (MRI) to determine the pathological changes occurring in the spinal cord, amount of cord compression and levels involved. These pathological spinal cord changes occuring at cellular level are observed as signal intensity (SI) changes on MRI [3]. After the initial descriptions by Takahashi et al. [4] on the prognostic significance of MRI SI changes, many authors have extensively examined the T2-weighted MRI SI changes to prove its predictive value [5]. However, there are authors who have refuted the above findings and stated that T2-weighted MRI SI changes have no predictive value $[6,7]$.

Various studies have examined different factors such as age, sex, duration of symptoms, presence of comorbidities, number of levels involved, spinal cord compression ratio on MRI, preoperative Japanese Orthopedic Association (JOA) score, MRI SI grading, pattern of SI change, levels of SI change, and SI ratio using both T1- and T2-weighted MR images, as well as their correlation with surgical outcome [8-10]. However, the predictive value of MRI SI changes remains unclear.

The lack of conclusive evidence could be explained by the fact that most of the studies published have only focused on either the presence or absence of intramedullary cord SI changes and very few of them have analyzed these SI changes either qualitatively by grading them, as in this study, or quantitatively by determining the SI ratios. Although recent studies have used SI change grading or SI change ratio for analyzing MRI SI changes, most are deficient in their methodology and data analysis because of the lack of uniformity with respect to data evaluation [11-13].

Only a few studies are prospective, have used the modified JOA (mJOA) score, and included patients irrespective of the approach used, and even fewer studies have attempted to determine the correlation between MRI SI changes and preoperative variables such as age, duration of symptoms, and baseline mJOA score [14]. Considering the above limitations, current recommendations regarding the prognostic value of T2-weighted MRI SI changes cannot be convincingly used in clinical practice [15].

This study prospectively collected data from patients who underwent surgery for cervical myeloradiculopathy by using anterior and or posterior surgical techniques. The aim of the study was to determine the correlation between intramedullary cord T2-weighted MRI SI change and preoperative factors such as age, duration of symptoms, and baseline mJOA score and to find the prognostic value of intramedullary cord T2-weighted MRI SI changes in predicting outcomes after surgery.

\section{Materials and Methods}

The study protocol was approved by the Institutional Review Board, and ethics committee approval was obtained. A total of 50 patients with cervical myeloradiculopathy referred to the spine clinic at a tertiary care institution from May 2012 to November 2013 were recruited. A wellwritten informed consent was obtained from each patient. After excluding four patients who were lost to followup, data from 46 patients (follow-up ratio, 92\%) were prospectively collected over a mean follow-up period of 1 year and analyzed. Patients aged $>18$ years who had a clinical diagnosis of myelopathy with or without radiculopathy were further investigated with specific X-rays and MRI cervical spine studies; the surgical candidates who gave consent were included in the study [16]. Patients with cervical cord compression on MRI who had no signs or symptoms of myelopathy were excluded, as were those who only had radiculopathy or who previously underwent a surgery for cervical myelopathy and/or radiculopathy. Patients with concomitant active infection, history of recent trauma, neoplasm or inflammatory spondyloarthropathy such as rheumatoid arthritis or ankylosing spondylitis were excluded.

MRIs performed within the last 6 weeks from the day of admission were accepted as fresh MRIs. MRI was performed either using the 1.5 tesla or higher configuration MRI machines. We used 5-mm-thick slices to identify the pathology level and disc herniation. Both T2- and T1weighted sagittal and axial MR images were obtained; however, in this study, only detailed evaluation of intramedullary cord T2-weighted sagittal MRI SI changes was performed.

\section{Interpretation of MRI SI changes}

Chen et al. [17] in 2001 provided a classification to grade the intramedullary cord T2-weighted MRI SI changes in patients with cervical myeloradiculopathy. They classified 
T2-weighted sagittal MR images for SI as follows: grade 0-no signal change (Fig. 1); grade 1-obscure/diffuse signal (Fig. 2); and grade 2-sharp focal signal of the same intensity as cerebrospinal fluid (Fig. 3). Classification of T2weighted MRI SI changes has been used in various studies to date $[18,19]$. A similar classification was used in this study, and all T2-weighted sagittal MR images were analyzed for intramedullary cord SI changes by an indepen-

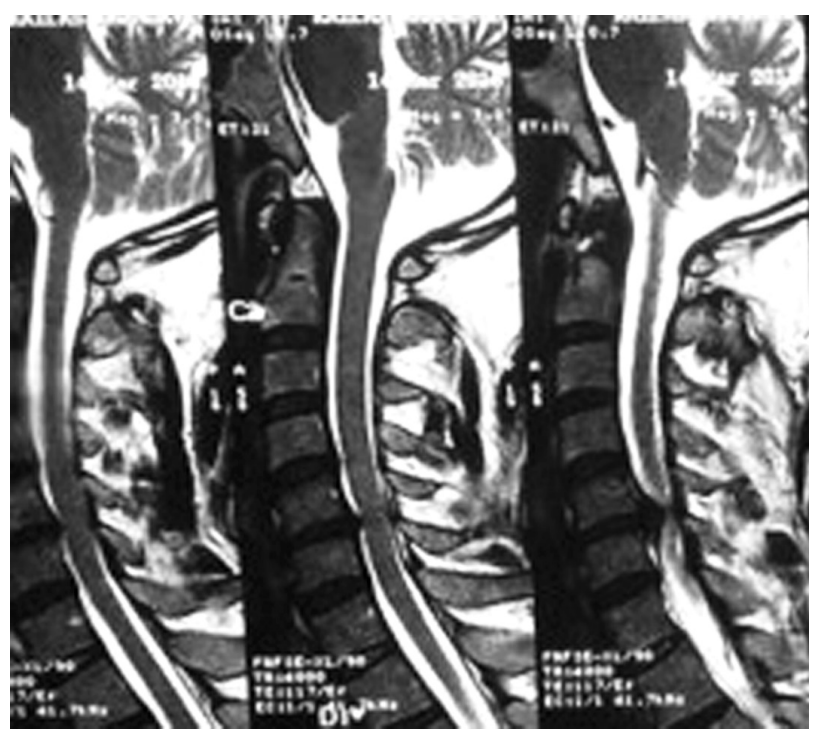

Fig. 1. Grade 0 signal intensity change.

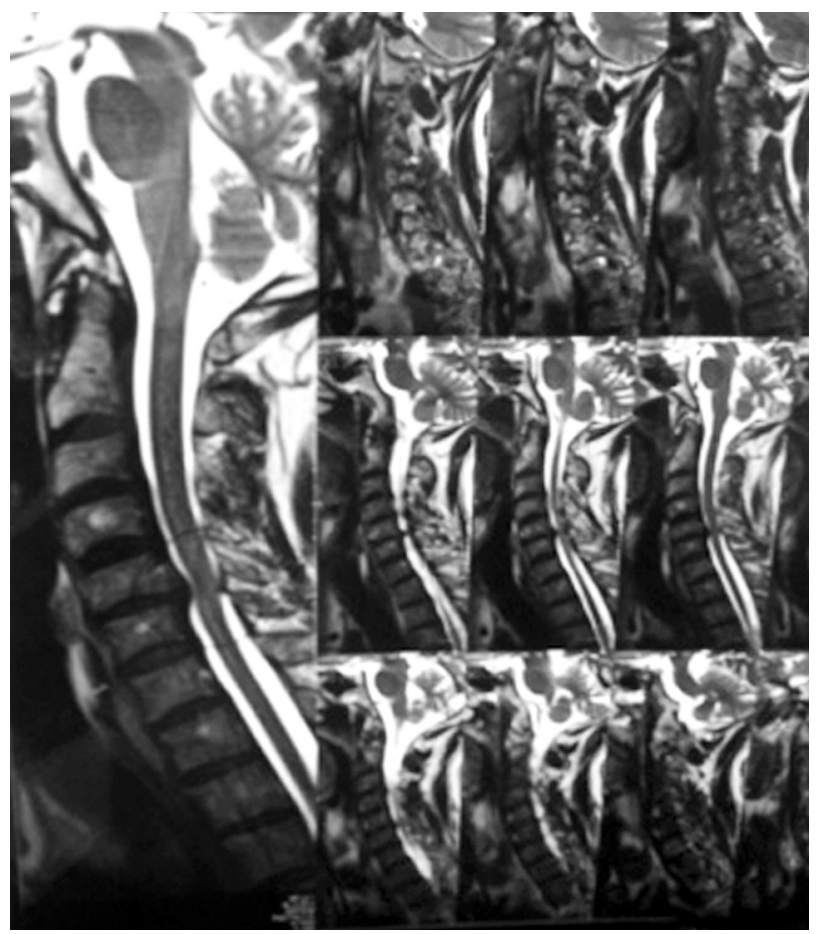

Fig. 2. Grade 1 signal intensity change. dent radiologist and a spine surgeon who were blinded to the patients' clinical and neurological status. There was an agreement for 44 of 46 grading of SI changes, and in the remaining two patients, the radiologists' opinion was taken as the final grading.

Fifteen patients who had single- or double-level prolapsed discs underwent surgery via the anterior approach, i.e., anterior cervical discectomy and fusion or anterior cervical corpectomy and fusion. The posterior approach, i.e., laminectomy with or without instrumentation and fusion was employed in 31 patients who had degenerative cervical spondylosis with canal stenosis or ossified posterior longitudinal ligament or had multilevel disc herniation. The indications and technique used were based on the recommendations made by several authors in their respective publications $[20,21]$.

\section{Outcome measures}

Preoperative assessments of the severity of cervical myeloradiculopathy were performed using the mJOA score. T2-weighted sagittal MRI SI changes of all patients were classified into three grades, and its correlation with factors such as age, duration of symptoms, preoperative mJOA score, and mJOA score at 1-year follow-up after surgery was analyzed. The 1-year follow-up signified a typical time

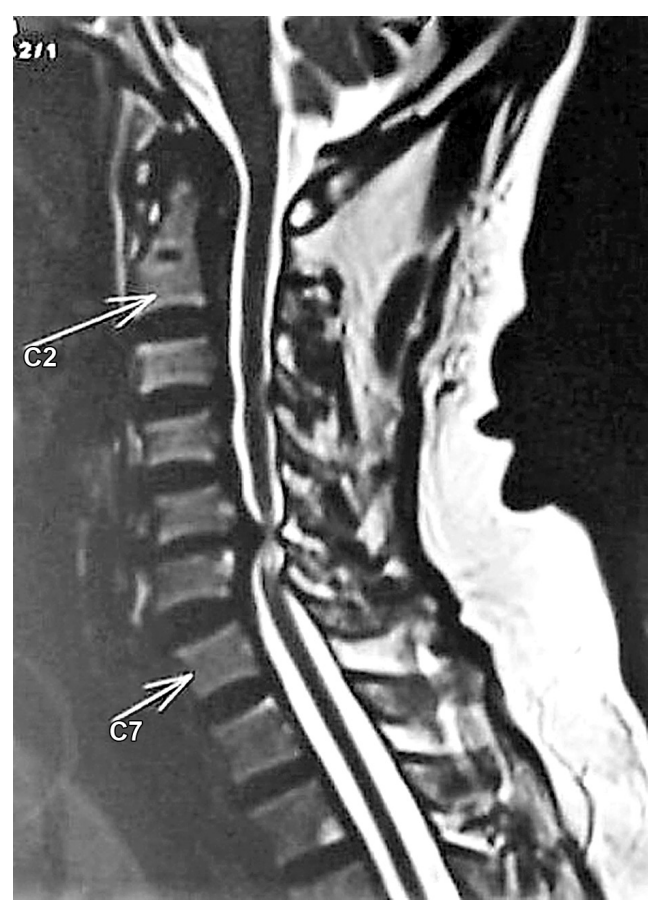

Fig. 3. Grade 2 signal intensity change. 
period of optimum recovery for degenerative cervical myeloradiculpathy [22].

\section{Statistical analysis}

Paired Students $t$-test was used for statistical testing of difference in mean values for comparing between preoperative and postoperative outcomes. A $p$-values of 0.05 were considered to be significant. Pearson's correlation was used to analyze the association between two variables. The analysis of variance (ANOVA) test was used to analyze multiple variables. Values were reported as means \pm standard deviation of the mean.

\section{Results}

The study included 43 men and three women; the mean age was $56.17 \pm 9.53$ years (range, $35-81$ years).

\section{Correlation between MRI grading and variables}

In this study, six of 46 patients had no intramedullary cord T2-weighted MRI SI changes. Twenty-one and 19 patients showed grade 1 and 2 changes, respectively. Table 1 shows analysis done using ANOVA test.

There was a significant correlation between $\mathrm{T} 2$-weighted MRI SI change grading, duration of symptoms ( $p=0.005)$, and mJOA score at 1 year $(p=0.017)$. There was no correlation among T2-weighted MRI SI changes, age at presentation, and baseline mJOA scores.

On further comparison of mJOA scores at 1 year after surgery, there was a significant correlation between patients with grade $0 / 1$ (no/obscure) SI changes (14.30 2.26 ; range, 9-17) and those with grade 2 (sharp/focal) SI changes $(12.47 \pm 2.20$; range, $8-17)(p=0.0187)$. Patients with grade $0 / 1$ SI changes fared better than those with grade 2 SI changes.
Postoperative complications occurred in four (8.69\%) of 46 patients examined. Two patients had vocal hoarseness that improved at later follow-ups without any intervention, and two patients had dural tears after posterior cervical surgery, which were repaired. No patients had local wound healing problems. No mortalities were recorded during the 1-year follow-up. No patients required revision surgery for recurrent symptoms.

\section{Discussion}

Various factors that affect patient outcomes after surgical treatment of cervical myeloradiculpoathy are well documented in the literature [8-10]. The huge number of recent studies on intramedullary cord T2-weighted MRI SI changes only highlights its importance and the dilemma the surgeon faces while commenting on its significance [11-13].

Intramedullary cord T2-weighted MRI SI changes represent various pathological changes in the cord [23]. It is a common practice in orthopedic clinics to examine T2weighted MR images and comment on the status of the spinal cord and nerve roots for treatment planning and prognostication.

There is controversy over the predictive value and the interpretation of MRI SI changes. High T2-weighted MRI SI changes represent reversible pathological changes to the intramedullary cord, whereas low T1-weighted SI changes represent irreversible damage to the intramedullary cord [23]. In contrast, Uchida et al. [24] proposed that SI changes are signs of severe chronic cord damage that may be irreversible.

Takahashi et al. [4] and Mehalic et al. [19] provided qualitative classifications of intramedullary cord T2weighted MRI SI changes, which were one of the earliest classifications published. Chen et al. [17] proposed an easier classification of SI grading and border pattern on

Table 1. Analysis done using ANOVA

\begin{tabular}{lcccc}
\multicolumn{1}{c}{ Variables } & MRI grade 0 & MRI grade 1 & MRI grade 2 & $p$-value \\
Age $(\mathrm{yr})$ & $51 \pm 10.47(38-61)$ & $54.48 \pm 5.96(42-65)$ & $59.68 \pm 11.49(35-81)$ & 0.079 \\
Duration of symptoms $(\mathrm{mo})$ & $5.33 \pm 0.86(4-6)$ & $10.81 \pm 6.645(2-24)$ & $16.06 \pm 8.363(4-36)$ & 0.005 \\
\hline Preoperative mJOA score & $11.33 \pm 2.16(8-14)$ & $11.57 \pm 2.71(7-15)$ & $9.84 \pm 2.32(6-16)$ & 0.090 \\
mJOA score at 1 year & $14.17 \pm 2.64(9-16)$ & $14.43 \pm 1.89(11-17)$ & $12.47 \pm 2.20(8-17)$ & 0.017 \\
\hline
\end{tabular}

ANOVA, analysis of variance; MRI, magnetic resonance imaging; mJOA, modified Japanese Orthopedic Association. 
T2-weighted imaging. Most studies, which used the above classifications, concluded that the sharp and intense SI correlated with poor neurological recovery [25-27], supporting our current findings.

Among the studies with contrasting results, Avadhani et al. [28] concluded that T2-weighted MRI SI changes (sharp or multisegmental) had no significant correlation with clinical outcomes, whereas Uchida et al. [24] recommended that T1-weighted MRI SI changes and not T2-weighted MRI SI changes were predictive of poor postoperative neurological recovery.

The other main study objective was to determine the predictive value of intramedullary cord T2-weighted MRI SI changes to prognosticate the outcome. There are few studies that have analyzed the association between T2weighted MRI SI changes and preoperative factors such as age, duration of symptoms, and baseline mJOA score. In this study, MRI grading for intramedullary cord SI changes did not correlate with age at presentation or preoperative baseline mJOA score but had significant correlation with the duration of symptoms and mJOA score at 1-year follow-up. These results are similar to those reported by Yukawa et al. [29] who concluded that patients who had a longer duration of illness had sharp SI changes and poorer outcomes, but they later reported another study in which they concluded that there was no correlation between T2weighted SI changes on postoperative follow-up MRIs and functional outcomes after surgery. We believe that T2weighted MRI SI changes represent some form of myelomalacia and gliosis that can only occur over an extended time period, and thus, is a chronic change. Thus, it would not be inaccurate to imply that a longer duration of symptoms would lead to sharp T2-weighted MRI SI changes [5,30]. T2-weighted MRI SI changes were significantly correlated with postoperative mJOA scores at 1-year follow-up. Patients with grade 0/1 SI changes fared better than those with grade 2 changes, implying that a sharp SI change in T2-weighted MRI can be considered as a predictor of poor functional recovery after surgery. These findings are similar to those published by Vedantam et al. in their recent review [26].

Thus, this study highlights and reestablishes the importance of classifying SI changes using T2-weighted sagittal MR images into absent/obscure, diffuse/sharp, and focal. Merely commenting on SI changes based on its presence or absence is not sufficient as more information regarding prognosis could be obtained by grouping the SI changes as above. Thus, we would like to emphasize on the importance of the association of sharp and focal T2-weighted MRI SI changes with poor prognosis, as was observed in our study, and issue a word of caution to physicians regarding the advice that they give to their patients and/or relatives about postoperative neurological recovery.

Wada et al. [5] and Fernandez de Rota et al. [31] suggested that involved levels of intramedullary cord SI changes should be considered while predicting outcomes. In this study, we found no correlation between the outcomes and the levels of cervical spinal cord showing intramedullary SI changes. SI changes are representatives of pathological changes in the cord, which is a continuous process, and may progress even after surgery [23]. Hence, to predict the outcomes based entirely on preoperative MRI would be fallacious. Avadhani et al. [28] and Uchida et al. [24] recently emphasized the importance of T1weighted SI changes while predicting outcomes for cervical myeloradiculopathy.

In this study, the T1-weighted MRI SI changes were not considered; however, we agree that a classification incorporating both T2- and T1-weighted MRI SI changes would be more useful in predicting the outcomes of postdecompression surgery, as well as a quantitative assessment such as SI ratio. This is a limitation of this study, and the other is the lack of long-term follow-ups. We would also like to perform postoperative MRI for patients on further follow-ups to substantiate our findings and help us provide clear guidelines while counseling patients with cervical myeloradiculopathy scheduled for surgery.

\section{Conclusions}

Patients with a longer duration of symptoms had higher grades of intramedullary cord T2-weighted MRI SI changes. Age and preoperative neurological status did not show a significant correlation with the existence of intramedullary cord T2-weighted MRI SI changes. However, it was observed that patients without or with mild and diffuse intramedullary cord T2-weighted MRI SI changes had better postoperative neurological recovery than those with sharp and focal cord SI changes.

\section{Conflict of Interest}

No potential conflict of interest relevant to this article was reported. 


\section{References}

1. Matz PG, Anderson PA, Holly LT, et al. The natural history of cervical spondylotic myelopathy. J Neurosurg Spine 2009;11:104-11.

2. Salvi FJ, Jones JC, Weigert BJ. The assessment of cervical myelopathy. Spine J 2006;6:182s-189s.

3. Matsuda Y, Miyazaki K, Tada K, et al. Increased MR signal intensity due to cervical myelopathy: analysis of 29 surgical cases. J Neurosurg 1991;74:887-92.

4. Takahashi M, Yamashita Y, Sakamoto Y, Kojima R. Chronic cervical cord compression: clinical significance of increased signal intensity on MR images. Radiology 1989;173:219-24.

5. Wada E, Yonenobu K, Suzuki S, Kanazawa A, Ochi T. Can intramedullary signal change on magnetic resonance imaging predict surgical outcome in cervical spondylotic myelopathy? Spine (Phila Pa 1976) 1999; 24:455-61.

6. Morio Y, Yamamoto K, Kuranobu K, Murata M, Tuda $\mathrm{K}$. Does increased signal intensity of the spinal cord on MR images due to cervical myelopathy predict prognosis? Arch Orthop Trauma Surg 1994;113:2549.

7. Naderi S, Ozgen S, Pamir MN, Ozek MM, Erzen C. Cervical spondylotic myelopathy: surgical results and factors affecting prognosis. Neurosurgery 1998;43:439.

8. Kohno K, Kumon Y, Oka Y, Matsui S, Ohue S, Sakaki S. Evaluation of prognostic factors following expansive laminoplasty for cervical spinal stenotic myelopathy. Surg Neurol 1997;48:237-45.

9. Alafifi T, Kern R, Fehlings M. Clinical and MRI predictors of outcome after surgical intervention for cervical spondylotic myelopathy. J Neuroimaging 2007;17:315-22.

10. Zhang YZ, Wang LF, Shen Y, Ding WY, Xu JX, He J. The effects of MRI signal intensity changes and clinical manifestations on prognosis after surgical intervention for cervical spondylotic myelopathy. Orthop Surg 2009;1:101-6.

11. Yone K, Sakou T, Yanase M, Ijiri K. Preoperative and postoperative magnetic resonance image evaluations of the spinal cord in cervical myelopathy. Spine (Phila Pa 1976) 1992;17:S388-92.

12. Fukushima T, Ikata T, Taoka Y, Takata S. Magnetic resonance imaging study on spinal cord plasticity in patients with cervical compression myelopathy. Spine (Phila Pa 1976) 1991;16:S534-8.

13. Morio $Y$, Teshima R, Nagashima H, Nawata K, Yamasaki D, Nanjo Y. Correlation between operative outcomes of cervical compression myelopathy and mri of the spinal cord. Spine (Phila Pa 1976) 2001;26: 1238-45.

14. Uchida K, Nakajima H, Sato R, et al. Multivariate analysis of the neurological outcome of surgery for cervical compressive myelopathy. J Orthop Sci 2005; 10:564-73.

15. Holly LT, Matz PG, Anderson PA, et al. Clinical prognostic indicators of surgical outcome in cervical spondylotic myelopathy. J Neurosurg Spine 2009; 11:112-8.

16. Montgomery DM, Brower RS. Cervical spondylotic myelopathy: clinical syndrome and natural history. Orthop Clin North Am 1992;23:487-93.

17. Chen CJ, Lyu RK, Lee ST, Wong YC, Wang LJ. Intramedullary high signal intensity on T2-weighted MR images in cervical spondylotic myelopathy: prediction of prognosis with type of intensity. Radiology 2001;221:789-94.

18. Yukawa Y, Kato F, Yoshihara H, Yanase M, Ito K. MR T2 image classification in cervical compression myelopathy: predictor of surgical outcomes. Spine (Phila Pa 1976) 2007;32:1675-8.

19. Mehalic TF, Pezzuti RT, Applebaum BI. Magnetic resonance imaging and cervical spondylotic myelopathy. Neurosurgery 1990;26:217-26.

20. Orr RD, Zdeblick TA. Cervical spondylotic myelopathy: approaches Approaches to surgical treatment. Clin Orthop Relat Res 1999;(359):58-66.

21. Bapat MR, Chaudhary K, Sharma A, Laheri V. Surgical approach to cervical spondylotic myelopathy on the basis of radiological patterns of compression: prospective analysis of 129 cases. Eur Spine J 2008; 17:1651-63.

22. Vitzthum HE, Dalitz K. Analysis of five specific scores for cervical spondylogenic myelopathy. Eur Spine J 2007;16:2096-103.

23. Ohshio I, Hatayama A, Kaneda K, Takahara M, Nagashima K. Correlation between histopathologic features and magnetic resonance images of spinal cord lesions. Spine (Phila Pa 1976) 1993;18:1140-9.

24. Uchida K, Nakajima H, Takeura N, et al. Prognostic value of changes in spinal cord signal intensity on 
magnetic resonance imaging in patients with cervical compressive myelopathy. Spine J 2014;14:1601-10.

25. Arvin B, Kalsi-Ryan S, Karpova A, et al. Postoperative magnetic resonance imaging can predict neurological recovery after surgery for cervical spondylotic myelopathy: a prospective study with blinded assessments. Neurosurgery 2011;69:362-8.

26. Vedantam A, Jonathan A, Rajshekhar V. Association of magnetic resonance imaging signal changes and outcome prediction after surgery for cervical spondylotic myelopathy. J Neurosurg Spine 2011;15:6606.

27. Ahn JS, Lee JK, Kim BK. Prognostic factors that affect the surgical outcome of the laminoplasty in cervical spondylotic myelopathy. Clin Orthop Surg 2010;2:98-104.

28. Avadhani A, Rajasekaran S, Shetty AP. Comparison of prognostic value of different MRI classifications of signal intensity change in cervical spondylotic myelopathy. Spine J 2010;10:475-85.

29. Yukawa Y, Kato F, Ito K, et al. Postoperative changes in spinal cord signal intensity in patients with cervical compression myelopathy: comparison between preoperative and postoperative magnetic resonance images. J Neurosurg Spine 2008;8:524-8.

30. Al-Mefty O, Harkey LH, Middleton TH, Smith RR, Fox JL. Myelopathic cervical spondylotic lesions demonstrated by magnetic resonance imaging. J Neurosurg 1988;68:217-22.

31. Fernandez de Rota JJ, Meschian S, Fernandez de Rota A, Urbano V, Baron M. Cervical spondylotic myelopathy due to chronic compression: the role of signal intensity changes in magnetic resonance images. J Neurosurg Spine 2007;6:17-22. 